\title{
Traditional Chinese Yuekou locks
}

\author{
Yang Zhang ${ }^{1}$ and Kuo-Hung Hsiao ${ }^{2}$ \\ ${ }^{1}$ College of Mechanical and Electrical Engineering, Beijing University of Chemical Technology, \\ 100029 Beijing, China \\ ${ }^{2}$ Collections and Research Division, National Science and Technology Museum, 80765 Kaohsiung, Taiwan \\ Correspondence: Yang Zhang (zhy@ mail.buct.edu.cn)
}

Received: 5 March 2020 - Revised: 21 August 2020 - Accepted: 27 September 2020 - Published: 5 November 2020

\begin{abstract}
In the Qing dynasty (1644-1911 CE), the town of Yuekou, in Hubei Province, was an important and renowned lock production center. Yuekou locks produced there won high acclaim for their use of quality materials. Transported via water routes, they were marketed in other provinces in China and other Southeast Asian countries. Even today, Yuekou locks are the favorites of antique collectors. After the introduction of the Western-style pin tumbler lock in the 1950s, the popularity of traditional Chinese locks began to decline, and they disappeared from the market. Receiving no attention from people, they were forgotten. From 2009 to 2016, the research team visited Yuekou eight times to conduct field research and interview surviving locksmiths and their descendants, achieving significant results. There are six main types of Yuekou locks, including combination locks, regular locks, pull-back locks, maze locks, multi-stage locks, and hidden-keyhole locks. The crafting of Yuekou locks is conducted in four stages: material preparation, molding, shaping, and assembling. The entire process is operated manually. This work introduces the historical development of Yuekou locks, summarizes the verbatim interviews, explores the production of Yuekou locks, and analyzes the types and structures of Yuekou locks.
\end{abstract}

\section{Introduction}

In Science and Civilisation in China, Joseph Needham (1965), a renowned historian of science and technology, mentions "The history of the progress of locksmiths' inventions in China remains extremely obscure, and everything is waiting to be done". Indeed, there have been a few thousand years of history regarding the making of locks in China. As the craft of lock making was passed down by masters to apprentices, although there were superb masters and refined crafts, their names are absent in history. Therefore, there is a general lack of literature regarding the making of locks in ancient times. However, since locks were widely used in different areas, they were handed down from ancient times. Among the existing objects, Yuekou locks are the epitome of ancient Chinese locks and provide a lot of valuable information. Therefore, this work focuses on the study of Yuekou locks. In the past few decades, related studies on traditional Chinese locks have begun to emerge. Hommel (1937) introduced a few commonly seen Chinese wooden tumbler locks and compared them with those wooden tumbler locks used in the countryside of France, Germany, Italy, and more. Needham (1965) introduced the development of Chinese and Western locks in history, briefly examining their mutual influences, and introduced the features of Chinese locks. Since 1990, Yan (2004) has formed collections of and conducted research on ancient Chinese locks and has systematically introduced the development and features of Chinese locks. Yan and Huang $(2000,2004 a, b)$ proposed the method of the design and making of ancient Chinese barbed-spring padlocks. Examining encyclopedias and dictionaries, they compared the implications of writings regarding locks in China and the West. With the topic of the internal structure of Chinese barbed-spring padlocks, Huang (2004) proposed a systematic design method of the keys, keyholes, and barbed springs' shapes and delineated the historical development of locks in China and the West. With reference to the materials and different periods of locks, $\mathrm{Li}$ (2007) categorized locks into four types - rope locks, wooden locks, metal locks, and electronic locks - to explain the development and applications of locks in China and the West. In 2012, NSTM (National Science 
and Technology Museum) held the "Under Keys and Locks Exhibition" and published a catalog to introduce the locks of ancient China and early Europe. All the exhibited items were illustrated with photos and brief explanations of their basic features and functions (Yan et al., 2012). Ye and Ye (2012) summarized ancient literature regarding the records of locks and introduced the development, craft, and making of locks and their cultural implications. With reference to three existing traditional Chinese maze locks, Shi et al. (2017) analyzed the structures of maze locks and discovered the process of inserting a key into a keyhole that can be regarded as the relation between two members and one joint. Hsiao (2017, 2018) examined puzzle locks with open keyholes and hidden keyholes to propose a method to analyze their structures systematically. Hsiao et al. (2019a, b) examined ancient Chinese Shanxi locks and puzzle locks to conduct a study on their development in history and analyze their structures. Shi et al. (2020) collected and summarized the actual relics of existing ancient Chinese wooden locks to propose a method of categorizing wooden locks. They also analyzed the structures and the processes of unlocking these wooden locks.

According to the unearthed relics and the circulation of actual objects, the barbed-spring padlock was used in locking doors, cabinets, chests, and more in China about 2000 years ago. As padlocks were used to lock chests, it ensured the safety of their contents, preventing them from being stolen during the transportation process. With the progress of time, barbed-spring padlocks became popular and were used in China extensively. In different regions, locksmiths designed and manufactured various kinds of locks to suit different needs. Although these locks were developed with similar structures, they were different in form and shape and made with different methods.

For a traditional Chinese padlock, there is a long shackle used for penetrating and fixing the two rings on the door or the box to prevent the door or box from being opened, as shown in Fig. 1a. Depending on different theories of design, padlocks can be categorized into two main types: barbedspring padlocks and combination padlocks. Between them, the barbed-spring padlock that can be opened with a key is the most representative one. In addition, it can be further divided into two kinds: open-keyhole locks and hiddenkeyhole locks depending on the visible features of the keyholes.

Figure 1b shows a simple open-keyhole padlock, which consists of four component members: a case, a bolt, barbed springs, and a key. On the case, there is a keyhole for inserting the key to enable the bolt's movement. A bolt consists of the shackle, the stem, and the end piece. The barbed springs are connected to the stem. The other side of the barbed springs touches the partition of the case to prevent it from being removed from the case, and this completes the locking function. The key head is designed in accordance with the position and form of the keyhole and the configuration of the barbed springs. The lock is opened by inserting the key until the key head presses the springs, and they are pushed through an opening in the partition. The bolt can now slide out of the case, freeing the shackle and opening the lock.

Locks with keyholes that cannot be seen at first glance are regarded as hidden-keyhole locks. In ancient times, locksmiths skillfully hid the keyholes using different means, requiring sliding a plate, pushing a button, revolving disks, and more. Therefore, there are many kinds of hidden-keyhole locks. The first step to unlock a hidden-keyhole lock is to find the keyhole. Figure 1c shows a kind of hidden-keyhole lock covered with a bottom plate. It consists of five members, including a case, a bolt, barbed springs, a bottom plate, and a key.

A traditional Chinese combination lock consists of three members: a case, three or more revolving disks, and a bolt. Figure 1d shows a combination lock with four revolving disks. The case is composed of an end piece and a shaft holding the revolving disks. The bolt includes another end piece, a shackle, and a stem with notches. As the shackle is inserted into the hole, and after the revolving disks are turned, it is locked.

Since the Qing dynasty, Yuekou locks have been famous for their diversity, high-quality materials, and refined artisanship and for being representative of traditional Chinese padlocks. However, due to the lack of literature, there is not any related study on crafting methods and types of Yuekou locks. Therefore, first, this work explains the characteristics of traditional Chinese padlocks. Then the historical development and the crafting method of Yuekou locks are discussed. Finally, the types and the internal structures of Yuekou locks are analyzed.

\section{Historical development of Yuekou locks}

Hubei Province is in the middle part of China, along the midstream of the Yangtze River. In Hubei, the internal river transportation was highly developed in the past, with prolific water resources, with more than 1000 rivers, big and small. Among them, the Yangtze River and Han River were the two major water transportation routes. In the 19th century, the villages in Hubei were only connected by footpaths. It made water transportation the most convenient and rapid means of transportation. The town of Yuekou was located in Hubei, along a bend of the Han River, with the convenience of water transportation and the geographical situation (Fig. 2). The Han River was the longest tributary of the Yangtze River. Around $175 \mathrm{~km}$ downstream from Yuekou, the Han River flowed into the Yangtze River at the port of Hankou. The water routes of the Yangtze River connected Yuekou with Hunan, Sichuan, and Guizhou in China. Since 1861, the city of Hankou had been a port opened to external trade. Likewise, the Yangtze River connected Hankou with the world. Goods from European and American countries and Japan, etc., were 


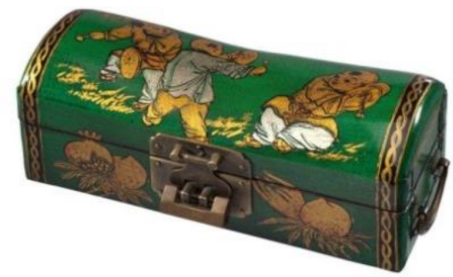

(a)
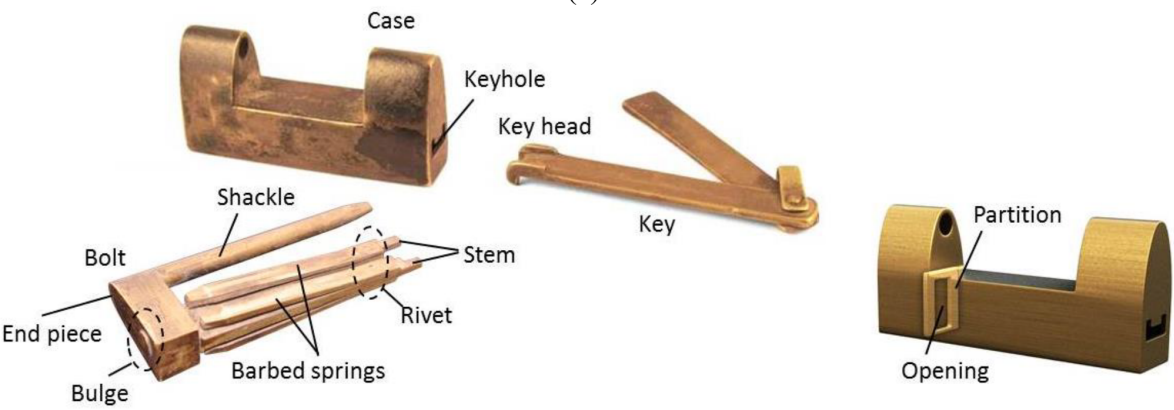

(b)
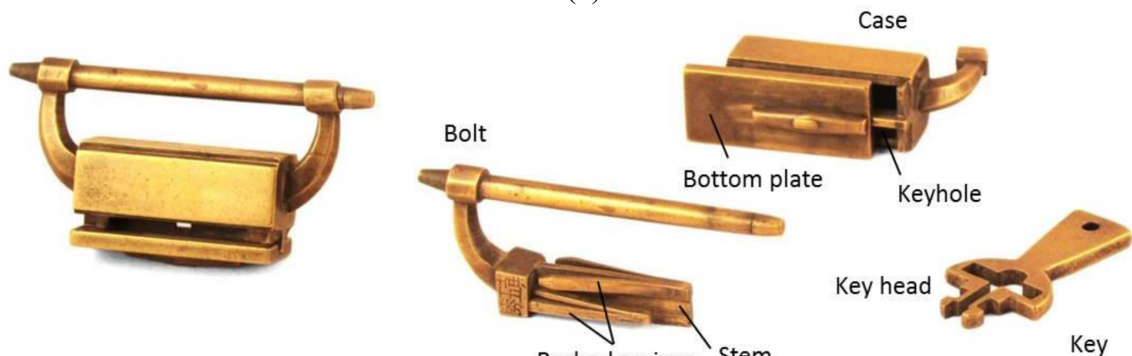

(c)

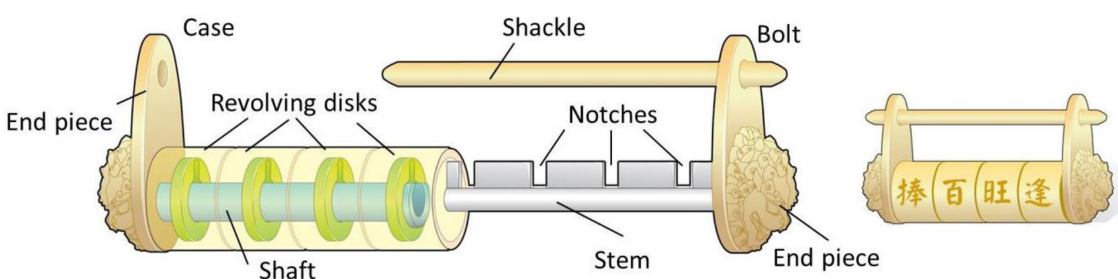

(d)

Figure 1. Traditional Chinese padlocks.

shipped to Hankou via sea routes. Then they were dispatched to different parts of China from Hankou.

According to Hubei Provincial Annals(1995; Fig. 3), in the late Qing dynasty (around 1850-1912), brass locks made in Yuekou became famous. In 1898, the manufacturing of brass locks prospered, with about 20 large and small lock shops forming a brass lock outlet at Sanchakou in Yuekou. The crafting of brass locks was conducted using several processes that were operated manually. The products were sold in China as well as Southeast Asian countries.

By 1938, there were still about 20 lock shops at Sanchakou in Yuekou (Fig. 4). Nevertheless, with the popularity and prolific sales of pin tumbler locks, traditional brass locks declined and disappeared from the market. After the 1970s,

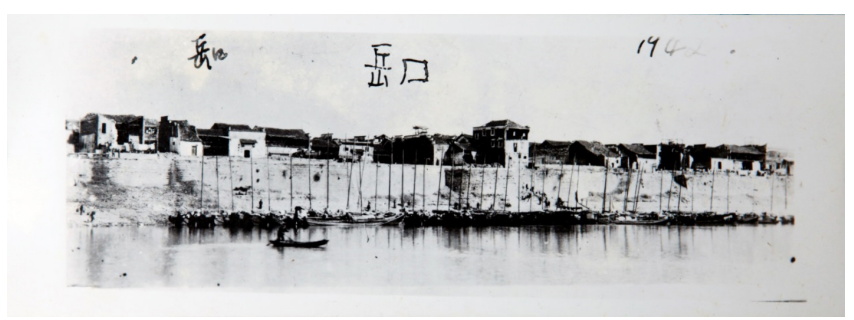

Figure 2. The embankment of the Han River at Yuekou in 1942 (collected by Yuxiang Chen 陳玉祥, 2010). There were many ships that docked in the port. It shows that the local shipping was very prosperous at the time. 


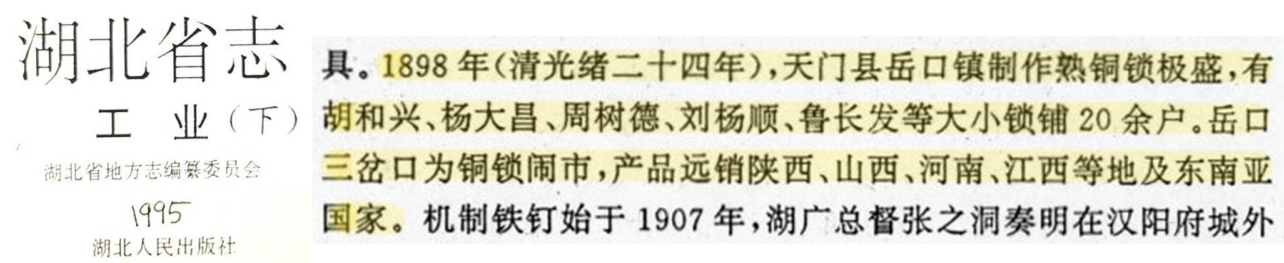

Figure 3. Quotes from Hubei Provincial Annals.

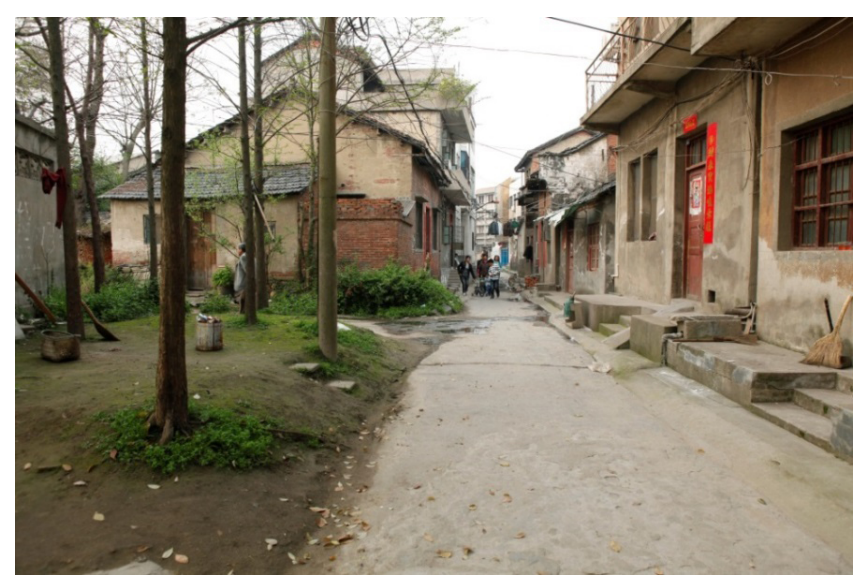

Figure 4. The original site of Sanchakou, Yuekou, in 2010 (photo by Niana Liu).

hardly any lock shops were left in Yuekou. To make a living, locksmiths changed the emphasis of their trade. Sometimes, they would repair old locks and make keys for old locks.

From 2009 to 2016, the research team visited Yuekou eight times on field investigations. We interviewed two locksmiths that made locks before and three sons or grandsons of former locksmiths. Among them, two locksmiths had come from Jiangxi and Shandong, escaping from disasters. They brought their skills to Yuekou and conducted lock businesses there. The other three were natives of Yuekou; learning from masters as apprentices and being self-taught, they developed the know-how of lock making and expanded their trade.

From the 1850 s to 1950 s, there were three major factors contributing to the prosperity of Yuekou locks. First, the quality of brass imported from Europe and America was much better than that produced in China. When high-quality brass was imported to Hankou, it could be transported to Yuekou quickly at a comparatively low price, providing locksmiths with quality materials. Second, the skill of lock making was excellent in Yuekou. In addition to the research and development on its own, excellent artisanship was imported from other provinces, inspiring better design and production methods. Third, locks produced in Yuekou could be transported to China and Southeast Asian countries quickly via sea routes. The quality locks, low transportation cost, and large market promoted the competitive advantages and uniqueness of Yuekou locks, which had become a major lock brand back then.

\section{The method of making Yuekou locks}

According to the investigation conducted in 2019, almost all the locksmiths in Yuekou were deceased. The craft of lock making in China is fading gradually. Fortunately, in April 2010, the research team interviewed a retired locksmith, Xiong Fa'er (1922-2012), and obtained first-hand materials, leaving valuable records regarding the making of Yuekou locks (Fig. 5). (Rongyu Su, Changping Zhang, and Wei Zhang conducted these interviews.) Mr. Xiong was the last locksmith in the village. According to his words, his grandfather and four brothers came from Jiangxi Province to escape disasters, bringing along the craft of lock making. Xiong's grandfather passed down the craft to Xiong's father, who taught Xiong the skills of lock making. From 14 years old, Xiong began to make locks. When he was 16, he could make locks independently. Xiong's father lived until he was in his 80s; however, he ceased to make locks when he was 60. It was because Xiong's father was blind from working near the furnace for a prolonged period. Generally speaking, locksmiths were either families or relatives. The craft was passed down to apprentices by masters. Most of the time, the craft was taught to sons by fathers. It was seldom taught to people not in the family. In 1959, Mr. Xiong's eyes became weak. In addition, the demand of traditional padlocks began to shrink, and Xiong therefore ceased to make locks.

According to the interview with Mr. Xiong and related data, the craft of making Yuekou locks can be summed up in four parts: keys, bolts, springs, and cases. Keys were made with the sand casting process in three major steps. First is the making of the key's model according to its shape. A bamboo plate with a thickness of about $2-3 \mathrm{~mm}$ is carved into the shape of the key for the making of the mold cavity (Fig. 6a). Second, as shown in Fig. 6b, the mold is made with two wooden blocks with a thickness of $70 \mathrm{~mm}$. The grooves are carved in the shape of the key between the two wooden blocks. A layer of dry charcoal ash is laid on the grooves for heat insulation and demolding. A layer of wet charcoal ash is filled in as green sand mold. The wet charcoal ash is used to make the key's shape. Then the bamboo key model is pressed into the wet charcoal ash. After pressing the two halves of 


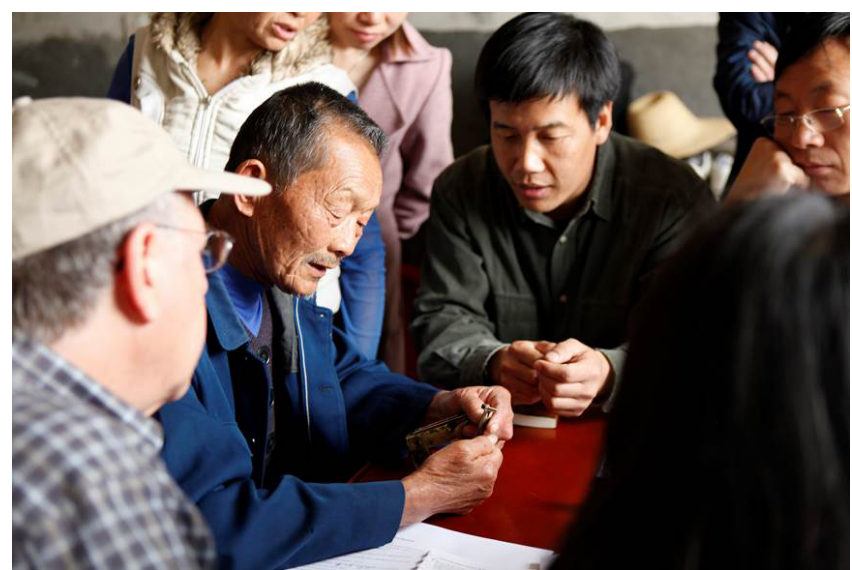

Figure 5. Interviewing Xiong Fa'er in 2010 (photo by Niana Liu).

the mold, the key is taken out. At that time, the cavity of the key is formed in the mold as shown in Fig. 6c. Then a sprue is set at the side of the mold. Third is the process of pouring. Pressing the mold firmly with the hands to tighten them (should be held at a certain slanting angle), the melted lead brass (at the approximate concentration of $60 \% \mathrm{Cu}, 35 \% \mathrm{Zn}$, $3 \% \mathrm{~Pb}, 1 \% \mathrm{Fe}$ ) is poured into the mold through the sprue, as shown in Fig. 6d. When it has cooled down, the mold can be opened, and the key can be removed. After trimming the key, it is completed and ready for use.

The process of bolt making is divided into two parts. First is the making of the shackle and end piece. It mainly adopts the sand casting process to form the shackle and end piece as one whole body. When making the mold, the end piece model is used to make the sand mold. Then a copper rod is inserted into the sand mold to make the cavity for the shackle. The stem is made with the same process. At the end of the stem, there is a cylindrical bulge. A round hole is pierced at an appropriate position on the end piece for the knocking in of the cylindrical bulge. The part of the cylindrical bulge that extends beyond the end piece is repeatedly hammered until flat to promote the strength of connection, as the bolt shown in Fig. 1b. The barbed spring is obtained by cutting a piece of brass $1.5 \mathrm{~mm}$ thick, according to a predesigned shape. The barbed spring and the stem are connected with a riveting process as well, as the barbed springs show in Fig. $1 b$.

The making of the case consists of two steps. The parts of the case are shown in Fig. 7a. Step 1 is to make the different parts separately. A brass plate of about $8 \mathrm{~mm}$ thick is cut and bent according to the size and shape of the case, as part $\mathrm{C}$ shows in Fig. 7b. Parts A and D are made by cutting the brass plate. Parts B, E, and F are made by cutting the brass plate to bring the external form first and then carving out the internal shape. Step 2 is to weld the parts together. Borax, called "moonstone" in Yuekou, is used as welding flux. Pliers are used to tighten up the parts, leaving no gaps. Borax is applied on the welding points, the parts are heated up, and the high temperature melts the borax, which flows along the connecting points to complete the welding process.

\section{Combination locks}

Yuekou locks were famous for their neat look and fine artisanship. According to the surviving relics, there are combination locks in the following types: three revolving disks, four revolving disks, and five revolving disks, as shown in Fig. 8. There are marks (most of them are Chinese characters) on each revolving disk. Inside each disk is a notch corresponding to one of the notches on the bolt's stem. Figure 8d is a 3-D illustration of the unlocking process of Fig. 8b. When unlocking the lock, each of the revolving disks should be turned (Fig. 8d1-d4). The characters or symbols on them should be turned to the right order as a code. Then, the notches in the disks will be aligned with the notches on the stem. The bolt can be taken out to complete the unlocking process (Fig. 8d5-d8).

Other than the code and number of revolving disks, the appearances, forms, and design styles are similar. On the sides of the lock are symmetrical eight-petal floral patterns as decoration. In addition, the rounded lower surfaces of each lock's end pieces are engraved with the characters 岳 (yue) and 口 (kou), for Yuekou, the production place. On the top of both ends of the locks are engravings of the lock shops' names. These are the major features of Yuekou combination locks. It is deduced that Yuekou locks were a famous brand at that time; therefore, the place and shops of production are engraved on the locks to promote value and brand identification, facilitating marketing.

\section{Barbed-spring locks}

The upper part of Yuekou barbed-spring locks has a smooth oval shape. Similar to the artisanship of Yuekou combination locks, Yuekou barbed-spring locks also exhibit excellent craftsmanship. Some of them have decorations on them, while others are simple and neat in form. Moreover, the end piece of most barbed-spring locks has an engraving with the name of the workshop or locksmith. Only a few of them are engraved with the name of the production place, Yuekou, on them. Nevertheless, according to the appearance and artisanship, Yuekou barbed-spring locks have salient features and symbols on them. They come in two types: open-keyhole locks and hidden-keyhole locks, introduced in the following subsections.

\subsection{Open-keyhole locks}

Yuekou's open-keyhole locks are rather diverse in form and are comparatively simple. According to the manner in which they are opened, they can be categorized into four types: regular locks, pull-back locks, maze locks, and multi-stage locks. In general, Yuekou's open-keyhole locks consist of 


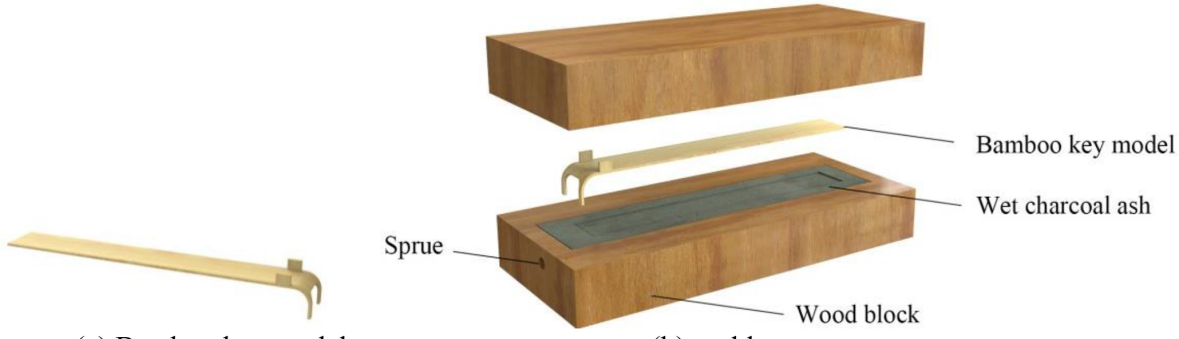

(a) Bamboo key model

(b) mold

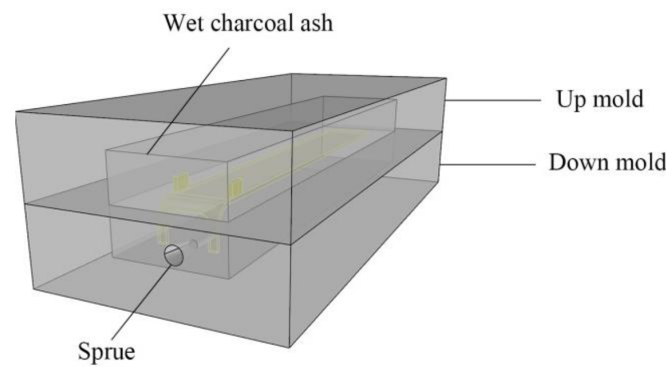

(c) Fitted together mold

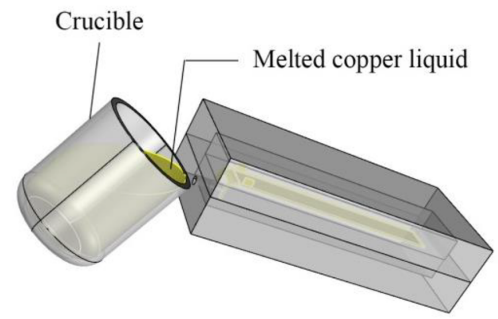

(d) Pouring process

Figure 6. Process of key making.

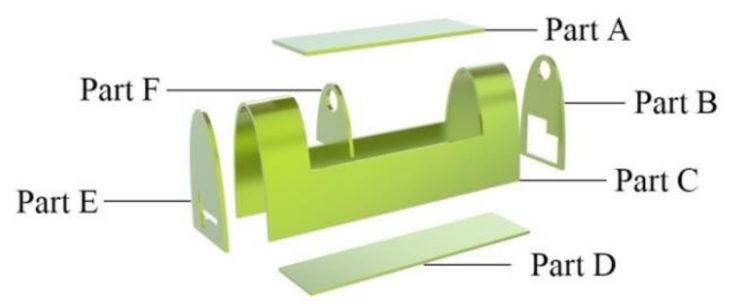

(a) Parts of the case
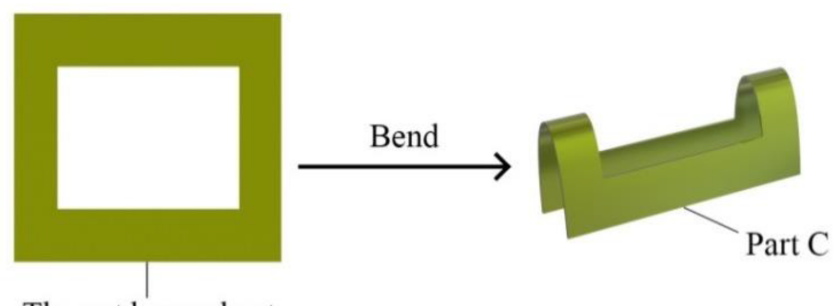

The cut brass sheet

(b) Process of making Part C

Figure 7. Process of case making.

four members: a case, a bolt, barbed springs, and a key. Figure 9a shows a kind of regular lock. With a special design, both upper and lower stems are riveted with two springs. In the middle, there is an obstacle rod to prevent the wrong key being inserted. Therefore, a 3-D hollow key must be used to press all the springs. With a simple structure, it is safe and secure. Moreover, this barbed-spring lock is one of the few that bears the engraving of the name of its production place on the end piece of the bolt. The inscription reads 岳口劉福太造
(Yuekou; made by Liu Futai). Figure 9b shows a 3-D illustration of the process of unlocking.

Regular barbed-spring locks can be opened by pushing the key forward to press the barbed springs. Nevertheless, Fig. 10a1 shows a pull-back lock, the spring of which is pressed by pulling the key backward to slide the bolt. The most special feature of this lock is that it uses a single spring connected directly to the bottom of the case. The spring blocks the stem to prevent the shackle from being pulled out to open the lock (Fig. 10a2). Figure 10b is a 3-D illustration of the process of unlocking. To open the lock, the key head is inserted horizontally into the keyhole until the key head is stopped by the stem (Fig. 10b1). Then the key is turned counterclockwise at $180^{\circ}$ to allow the key head to face down (Fig. 10b2). As the key head presses the spring attached to the bottom of the case, it releases the stem (Fig. 10b3). The key is pulled back and the bolt is slid out to complete the unlocking process (Fig. 10b4).

Maze locks must be opened by placing the key at a particular position and moving it in a certain direction to smoothly insert the key head into the keyhole. As the process of unlocking is like walking in a maze, it is called likewise. Designed in such a manner, even if a person holds the key and can see the keyhole clearly, it is still difficult for them to insert the key into the keyhole. Yuekou maze locks are diverse in form and shape. They can be subcategorized into three kinds types, according to the first step of inserting the key head into the keyhole: turning, sliding, and twisting, as shown in Fig. 11a-c.

Figure 11a is provided as an example to illustrate the opening process. Three steps are necessary to insert the key head into the keyhole, and there are six steps in total in the opening 


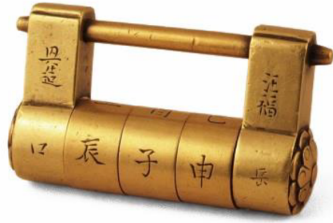

(a)

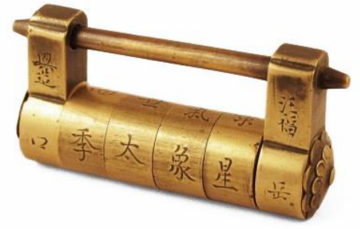

(b)

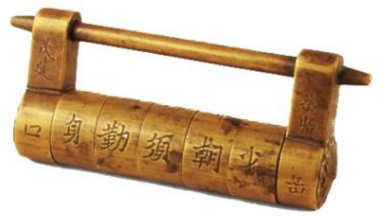

(c)
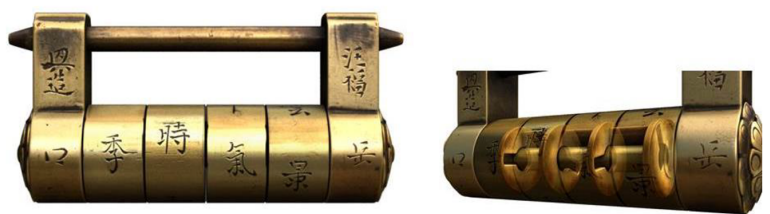

(d)

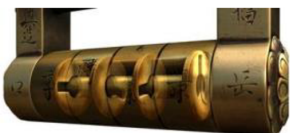

$\left(d_{1}\right)$

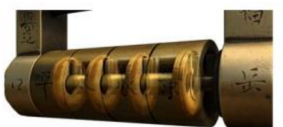

$\left(d_{5}\right)$

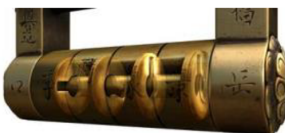

$\left(d_{2}\right)$

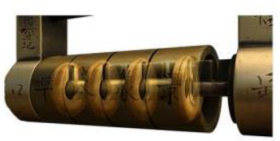

$\left(d_{6}\right)$

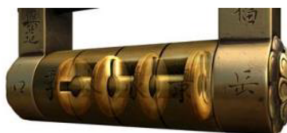

$\left(d_{3}\right)$

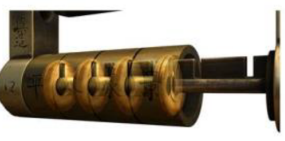

$\left(d_{7}\right)$

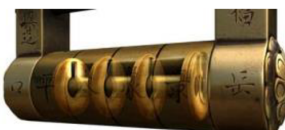

$\left(\mathrm{d}_{4}\right)$

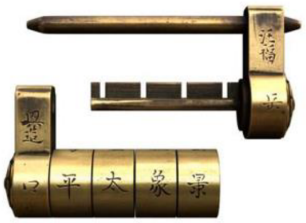

$\left(d_{8}\right)$

Figure 8. Combination lock.

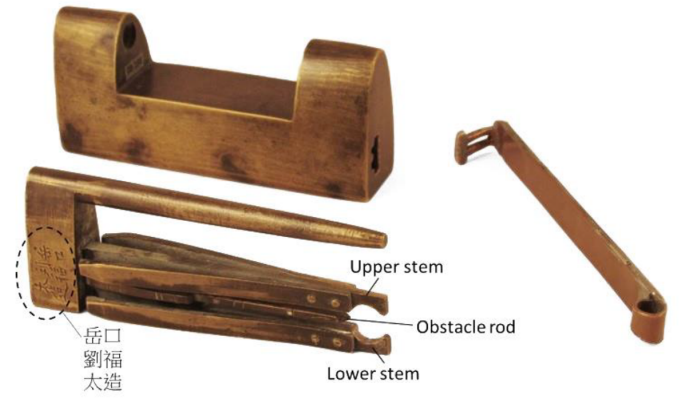

(a)

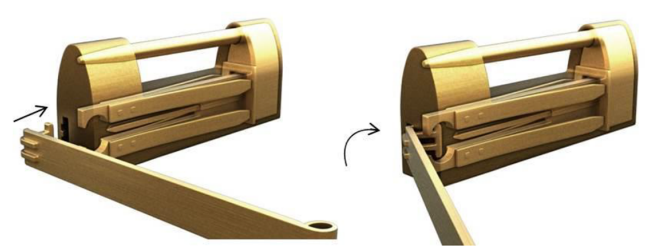

$\left(b_{1}\right)$

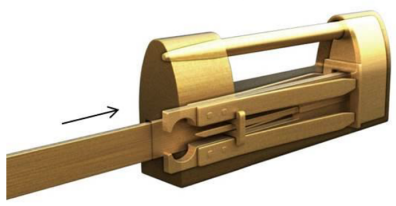

$\left(b_{4}\right)$ $\left(b_{2}\right)$

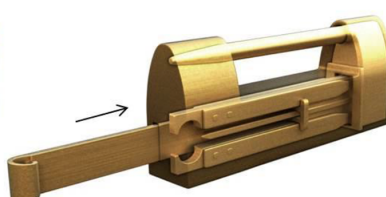

(b)

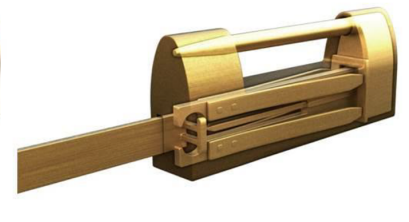

$\left(b_{3}\right)$

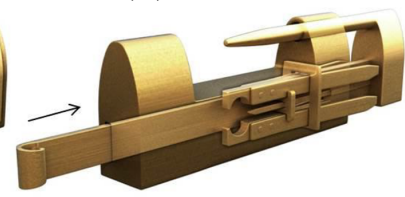

$\left(b_{6}\right)$

Figure 9. Regular lock. 


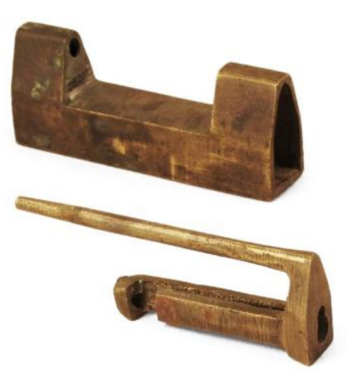

$\left(a_{1}\right)$

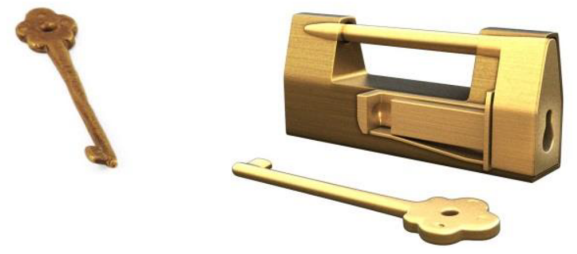

$\left(a_{2}\right)$
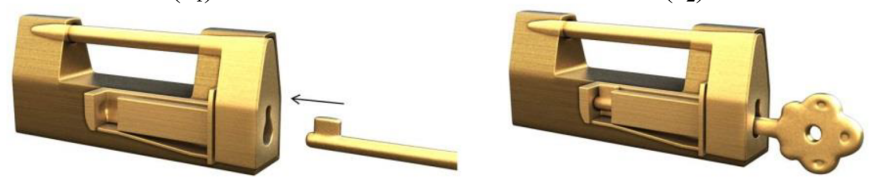

$\left(b_{1}\right)$

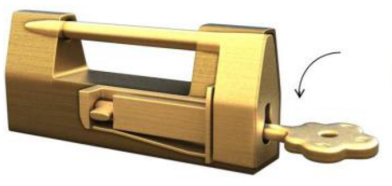

$\left(b_{2}\right)$

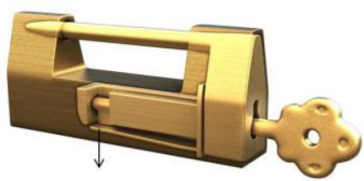

$\left(b_{3}\right)$

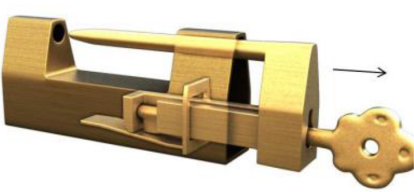

$\left(b_{4}\right)$

Figure 10. Pull-back lock.

process. The first step is to place the key head horizontally over the keyhole (Fig. 11d1) and turn the key about the negative $z$ axis with respect to the case (Fig. 11d2). The second step is to turn the key about the positive $x$ axis with respect to the case (Fig. 11d3). Third, the key is turned about the negative $z$ axis with respect to the case (Fig. 11d4). The fourth step is to push the key to press the barbed springs in order to allow them to pass the opening of the partition (Fig. 11d5). When the barbed springs enter the opening of the partition, the bolt is moved by the pressing of the key (Fig. 11d6). Finally, the bolt is pushed completely out of the case, and the lock is opened (Fig. 11d7).

According to the lengths of the barbed springs, it is possible to slide the bolt out of the case all at once or in several incremental stages. The locks shown in Figs. 1b-c and 9-11 are one-stage locks for which the key heads can press all the barbed springs simultaneously, and the bolts can be slid from the case completely. They are one-stage locks. Figure 12a1 shows a two-stage lock with two keys and one keyhole for which the bolt needs to be slid from the case in two stages. It consists of six members, including the case, the bolt, barbed springs 1 , barbed springs 2 , key 1 , and key 2 . To open the lock, each stage has three steps, including inserting the key, pressing the barbed springs, and sliding the bolt. In the first stage, key 1 is inserted with the key head facing down into the keyhole to press barbed springs 1 . When the barbed springs 1 enter the opening of the partition, the bolt can be slid out a little bit. When barbed springs 2 touch the partition, the bolt is stopped from moving. Key 1 is taken out to complete the first stage. In the second stage, key 2 is inserted with the key head facing up into the keyhole to press barbed springs 2 .
When the barbed springs 2 enter the opening of the partition, the bolt can be slid out of the case to open the lock.

Since the most common type of damage is the barbed springs in Yuekou locks, the variation of friction force of the spring is analyzed. First, taking the outline drawing of the red spring shown in Fig. 12a2 for curve fitting, the following deflection $\delta$ function can be obtained as shown in Fig. 12b:

$$
\left\{\begin{array}{c}
\delta=0(0 \leq x \leq 11 \mathrm{~mm}) \\
\delta=0.00104 x^{2}+0.0257 x-0.40854(x \geq 11 \mathrm{~mm})
\end{array} .\right.
$$

When the key enters and presses the spring, the normal pressure is

$P=3 E I \delta /\left(x-x_{0}\right)^{3}$,

where $x_{0}$ is the equivalent constraint position of the spring. The deflection is

$0.00104 x^{2}+0.0257 x-0.40854 \delta=$.

Suppose the friction coefficient is $\mu$, the friction force is

$0.00104 x^{2}+0.0257 x-0.40854 F=\mu P=\left(\frac{3 \mu E I}{\left(x-x_{0}\right)^{3}}\right)$.

The friction force is dimensionless using the following formula:

$F^{*}=\frac{F L^{2}}{3 \mu E I}$,

which obtains

$0.00104 x^{2}+0.0257 x-0.40854 F^{*}=\frac{L^{2}}{\left(x-x_{0}\right)^{3}}$. 


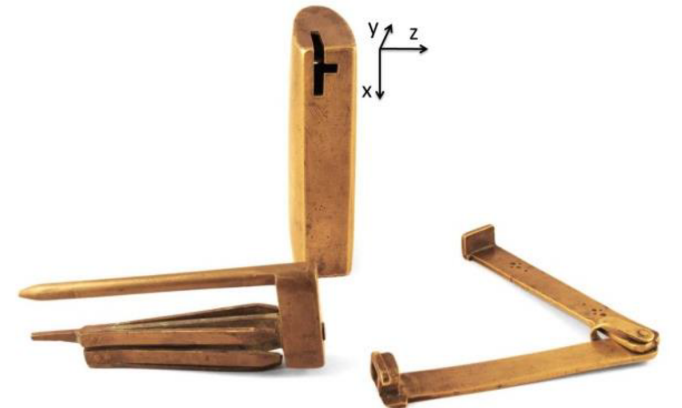

(a) Turn to insert the key

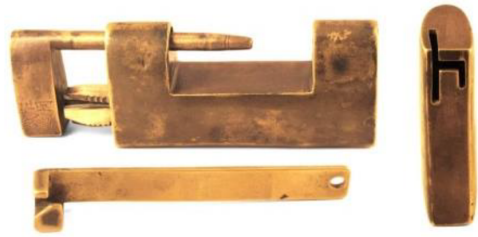

(b) Slide to insert the key

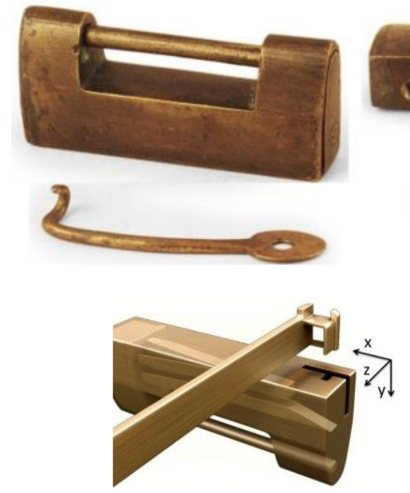

$\left(d_{1}\right)$

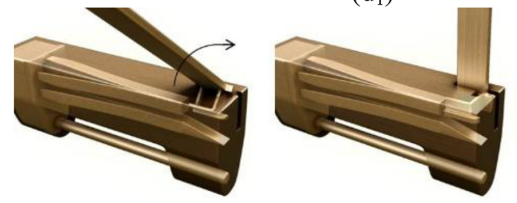

$\left(d_{3}\right)$

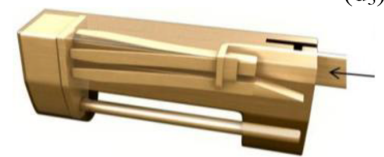

$\left(d_{5}\right)$

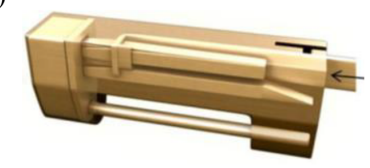

$\left(d_{6}\right)$

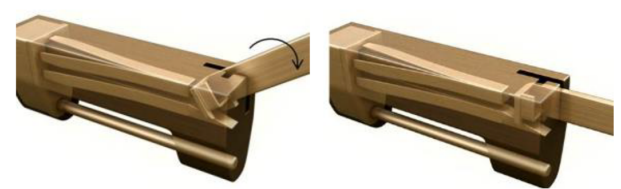

$\left(d_{4}\right)$

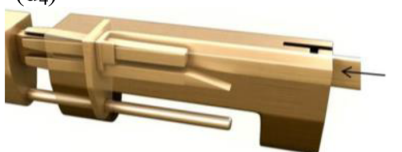

$\left(d_{7}\right)$

Figure 11. Maze locks.

The corresponding change relationship is shown in Fig. 12c.

It is shown in Fig. 12c that the maximum friction force appears at about $15 \mathrm{~mm}$ if it takes $x_{0}=5 \mathrm{~mm}$. As the position of the key restraint spring gets closer, the friction force becomes smaller. Similarly, for other springs, the corresponding friction analysis can be carried out using the same method.

\subsection{Hidden-keyhole locks}

Yuekou's hidden-keyhole locks exhibit superb craftsmanship. A sword pattern is set in the middle of the bottom plate, and there is an end button on each end plate. Some buttons are designed as floral shapes. The keyhole is ingeniously concealed as shown in Fig. 13. Figure 13b shows a hiddenkeyhole lock for which the key can be inserted at the bottom of the lock. The end piece is engraved with the characters 汪福興造 (made by Wang Fuxing). There are two buttons, one movable and the other fixed. The long spring is located on the top of the stem connected with the movable button. The short spring is below the stem. The lock has seven parts: a case, a bolt, a long spring with moveable button, a short spring, an end plate, a bottom plate, and a key. When it is locked, the long spring is in contact with the partition inside the case to hold the bolt. To open the lock, there are 10 steps. The first step is to push down the moveable button along the negative $y$ axis to free the long spring trapped by the partition inside the case (Fig. 13c1). Step 2 is to slide the bolt along the positive $x$ axis a little bit (Fig. 13c2). When the short spring touches the partition, the bolt is stopped from moving. Since a part of the bolt has slid a little bit, the end plate is released from the shackle and can be moved. Step 3 is to turn the end plate about the positive $x$ axis to expose part of the keyhole (Fig. 13c3). Step 4 is to slide the bottom plate along the positive $x$ axis to reveal the keyhole completely (Fig. 12c4). In addition to concealing the keyhole, the design also includes 


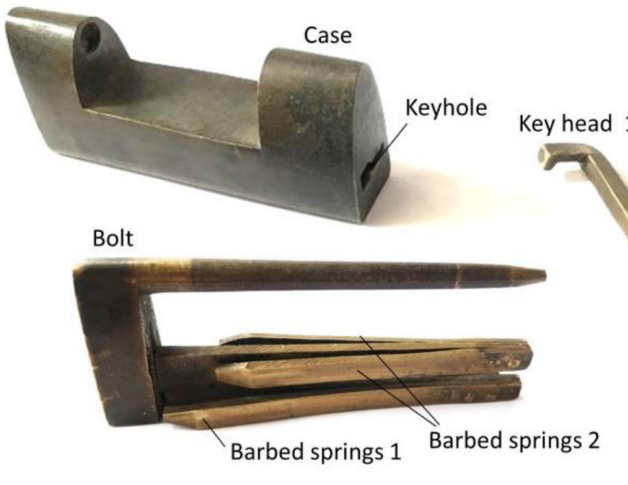

$\left(a_{1}\right)$
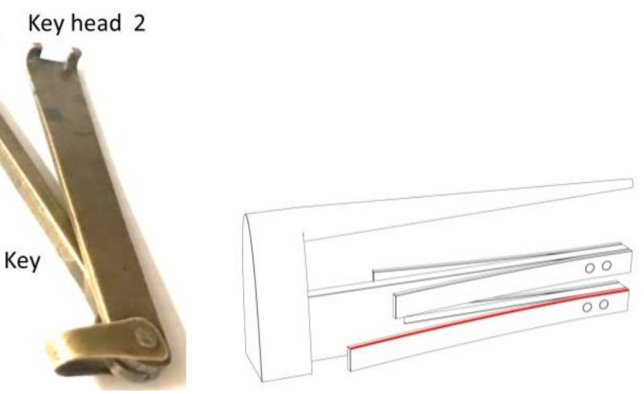

$\left(a_{2}\right)$

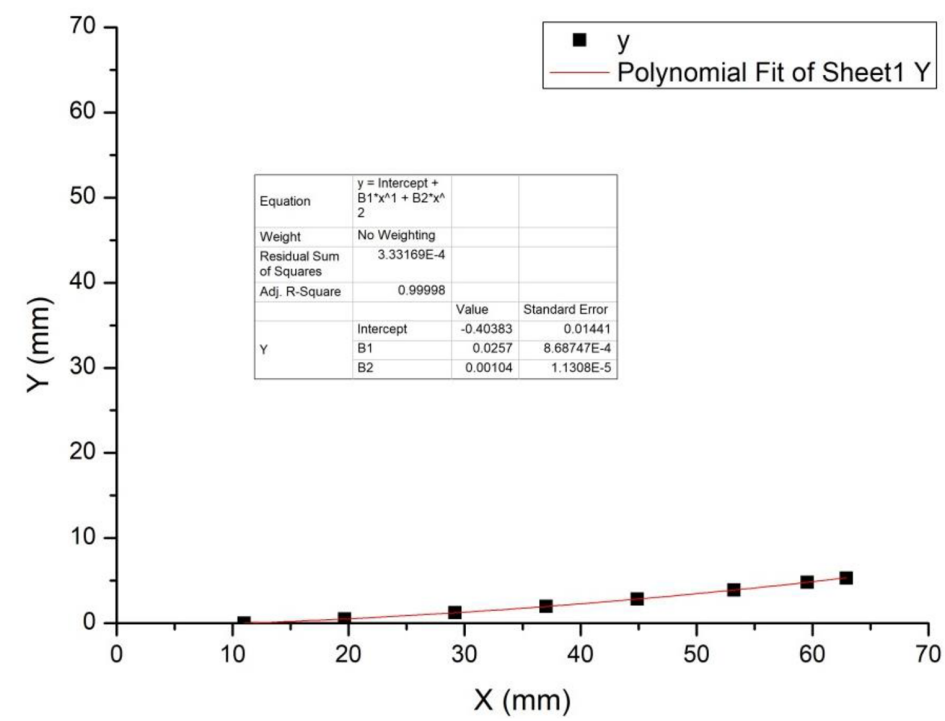

(b) Deflection curve fitting

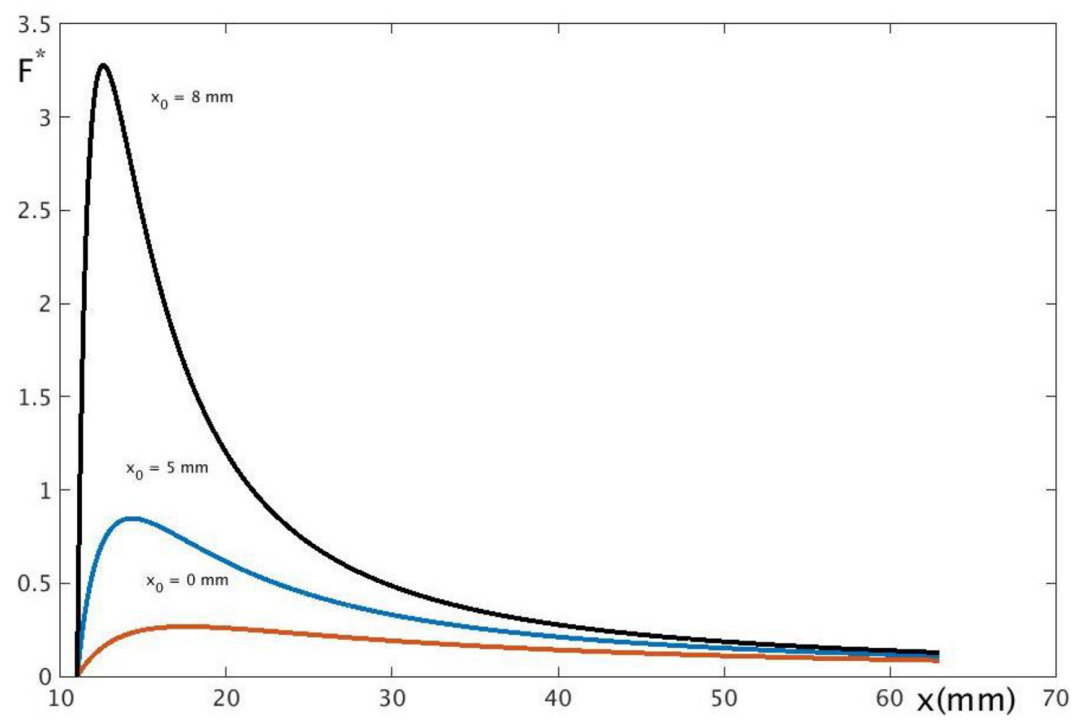

(c) The law of friction changing with distance

Figure 12. Two-stage lock. 


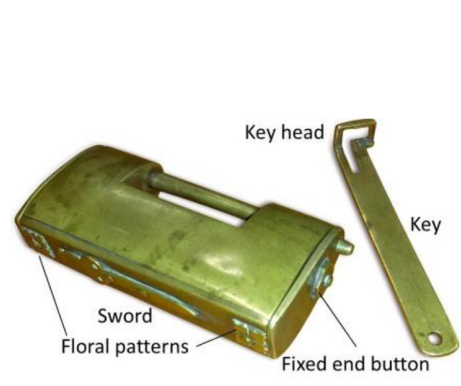

(a)

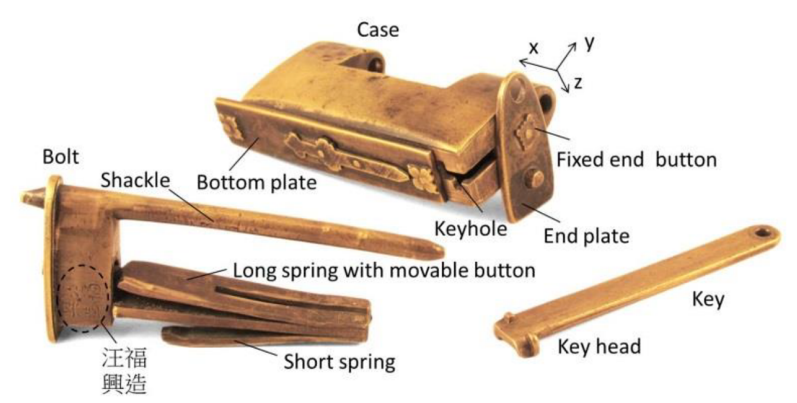

(b)

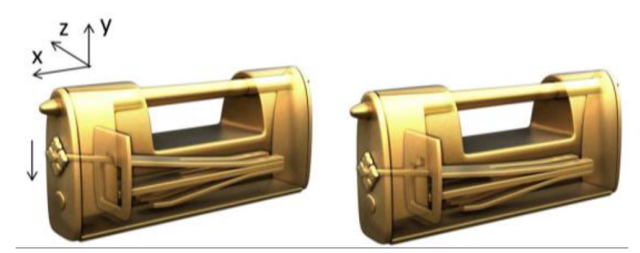

$\left(c_{1}\right)$

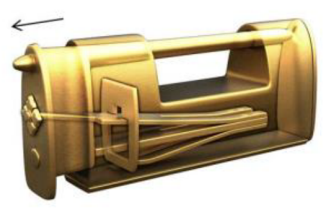

$\left(c_{2}\right)$

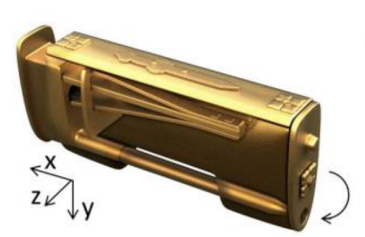

$\left(c_{3}\right)$

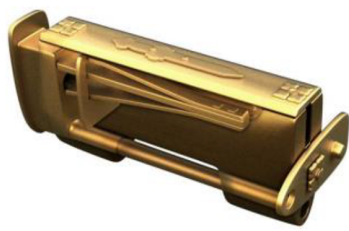

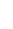
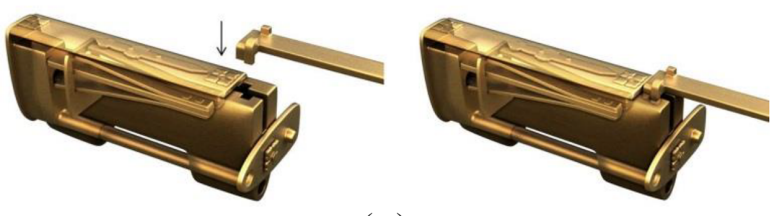

$\left(\mathrm{c}_{5}\right)$

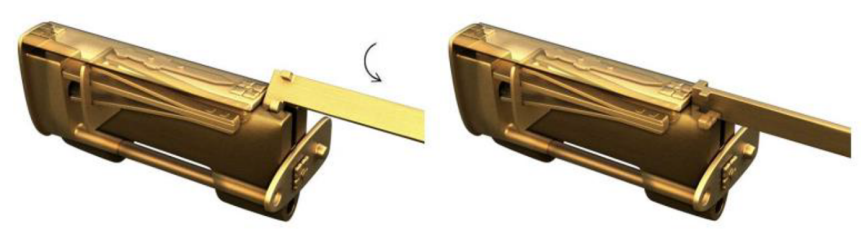

$\left(\mathrm{c}_{6}\right)$

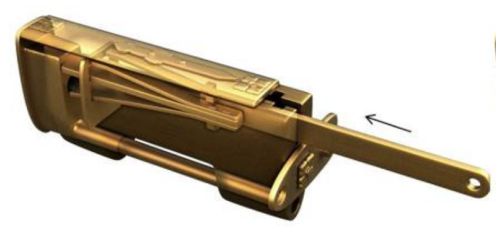

$\left(c_{8}\right)$

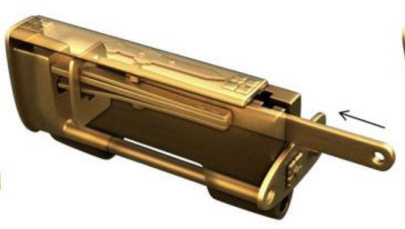

$\left(c_{9}\right)$

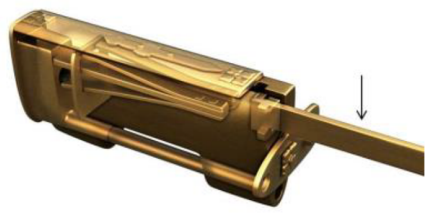

$\left(c_{7}\right)$

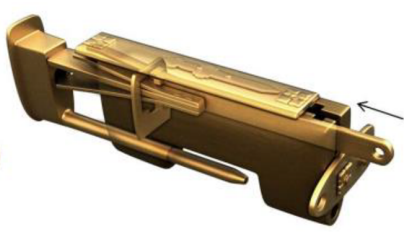

$\left(\mathrm{c}_{10}\right)$

Figure 13. Hidden-keyhole locks.

maze entries. Step 5 is to aim the key head at the keyhole horizontally, and slide and insert the key along the positive $y$ axis (Fig. 13c5). Step 6 is to turn the key head about the negative $x$ axis (Fig. 13c6). Step 7 is to slide the key head along the positive $y$ axis (Fig. 13c7). After the key has been moved to press the springs, the remainder of the opening process is similar to steps 4-6 of the maze lock of Sect. 5.1, as shown in Fig. 13c8-c10. After finding the keyhole, the other troubling issue is how to insert the key head into the keyhole. It is 
comparatively complicated to open locks with such intricate mechanisms.

\section{Conclusions}

Humans have been using locks for thousands of years. The development of locks is closely related to the craft, skills, materials, and tools of the era. As padlocks are convenient to use and can be circulated freely with goods, depending on the local environment in different districts in China, padlocks with different features have been developed. According to the theories of the design, they can mainly be categorized into two types: combination padlocks and barbed-spring padlocks. Due to the special geographical location and conditions for production in Yuekou, since the Qing dynasty, it was an area famous for producing padlocks. In addition to being sold in different provinces in China, they were marketed to different Southeast Asian countries, being a representative brand of traditional padlocks. This is the first research paper regarding Yuekou locks in China. Having conducted several field research studies, interviewed locksmiths and related personnel, and analyzed the structures of the antique locks, the historical development, production, lock types, mechanical structures, and unlocking process of Yuekou locks are proposed. Being first-hand material regarding the study of Yuekou locks, the paper can serve as a reference for scholars and experts interested in this area. It is expected to motivate more research on traditional Chinese locks.

Data availability. The data are available upon request from the corresponding author.

Author contributions. YZ was the lead author and performed and directed the analysis of the model represented in the paper. $\mathrm{KHH}$ initially structured the paper, made contributions to presentation approaches for the mechanisms, and provided reviews of the paper.

Competing interests. The authors declare that they have no conflict of interest.

Acknowledgements. The authors are grateful to the Ministry of Science and Technology (Taipei, Taiwan) under grant MOST 1092622-E-359-001-CC2 for the financial support of this work. Support from the Ancient Chinese Machinery Cultural Foundation (Tainan, Taiwan) and the Classical Chinese Puzzle Foundation (Berkeley, USA) are greatly appreciated. The authors would like to thank Wei Zhang, Peter Rasmussen, Rongyu Su, Niana Liu, and Changping Zhang, who conducted the long interview with Xiong Fa'er, and the collector Zhi Yin (尹志), who has been of continuous assistance.
Financial support. This research has been supported by the Ministry of Science and Technology (Taipei, Taiwan) (grant no. MOST 108-2622-E-359-001-CC2).

Review statement. This paper was edited by Guowu Wei and reviewed by two anonymous referees.

\section{References}

Hommel, R. P.: China at Work: An Illustrated Record of the Primitive Industries of China's Masses, Whose Life is Toil, and thus an Account of Chinese Civilization, 1st Edn., John Day Company, New York, USA, 294-304, 1937.

Hsiao, K. H.: On the Structural Analysis of Open-keyhole Puzzle Locks in Ancient China, Mech. Mach. Theory, 118, 168-179, 2017.

Hsiao, K. H.: Structural Analysis of Traditional Chinese Hiddenkeyhole Padlocks, Mech. Sci., 9, 189-199, 2018.

Hsiao, K. H., Zhang, Y., Shi, K., Chen, Y. H., and An, H.: Ancient Chinese Puzzle Locks, Robotics and Mechatronics, 1st Edn., edited by: Kuo, C. H., Lin, P. C., Essomba T., and Chen, G. C., Springer, Switzerland, 494-501, 2019a.

Hsiao, K. H., Zhang, Y., Lin, J. L., Huang, J. F., and An, H.: A Study on Ancient Chinese Shanxi Locks, Advances in Mechanism and Machine Science, edited by: Tadeusz, U., 1st Edn., Springer, Switzerland, 1179-1186, 2019b.

Huang, H. H.: Mechanism Design of Ancient Chinese Splittingspring Padlocks, Ph. D., National Cheng Kung University, Tainan, Taiwan, 14-45, 2004 (in Chinese).

Hubei Provincial Annals: Hubei Local Annals Editorial Board Eds., Hubei People's Press, Wuhan, China, 1332-1333, 1995 (in Chinese).

Li, W. S.: Illustration of Lock History, Shanghai Bookstore Press, Shanghai, China, 12-179, 2007 (in Chinese).

Needham, J.: Science and Civilisation in China, Vol. 5, Part 7, Cambridge University Press, Cambridge, UK, 236-242, 1965.

Shi, K., Zhang, Y., Lin, J. L., and Hsiao, K. H.: Ancient Chinese Maze Locks, T. Can. Soc. Mech. Eng., 41, 433-441, 2017.

Shi, K., Hsiao, K. H., Zhao, Y., Huang, C. F., and Xiong, W. Y.: Structural Analysis of Ancient Chinese Wooden Locks, Mech. Mach. Theory, 146, 1-13, 2020.

Yan, H. S.: The Beauty of Ancient Chinese Locks, 2nd Edn., Ancient Chinese Machinery Cultural Foundation, Tainan, Taiwan, 14-117, 2004.

Yan, H. S. and Huang, H. H.: On the Spring Configurations of Ancient Chinese Locks, Proceedings of 2000 HMM International Symposium on History of Machines and Mechanisms, edited by: Ceccarelli, M., Casino, Italy, 87-92, 2000.

Yan, H. S. and Huang, H. H.: A Study on Western and Chinese Locks Based on Encyclopedias and Dictionaries, Proceedings of 2004 HMM International Symposium on History of Machines and Mechanisms, edited by: Ceccarelli, M., Casino, Italy, 41-55, 2004a.

Yan, H. S. and Huang, H. H.: Design Consideration of Ancient Chinese Padlocks with Spring Mechanisms, Mech. Mach. Theory, 39, 797-810, 2004b. 
Yan, H. S., Li, J. C., and Hsiao, K. H.: Under Keys \& Locks, National Science and Technology Museum, Kaohsiung, Taiwan, 48-126, 2012.

Ye, D. B. and Ye, L. J.: Cultural History of Chinese Locks, Intellectual Property Publishing House, Beijing, China, 3-97, 2012 (in Chinese). 